\title{
Nanowires for Energy Applications: Fundamental Growth Studies
}

\author{
A. F. Marshall, I. A. Goldthorpe, M. Koto, P.C. McIntyre, J. Zhu, H. Peng, D.M. Barnett, W.D. Nix, \\ and Y. Cui
}

Geballe Laboratory for Advanced Materials and Department of Materials Science and Engineering, Stanford University, Stanford, CA 94305

Nanowire (NW) structures are being extensively investigated as components for energy devices such as solar cells [1] and batteries [2]. The large aspect ratio of NWs makes them ideal candidates for maximizing surface reactions, minimizing carrier transport distances, fabricating semi-transparent contact layers, and other device optimization pathways. Although methods for growing NWs are well established, and much current research is focused on device fabrication and characterization, much remains unknown about fundamental aspects of the nucleation and growth mechanisms, and the effect of such parameters as different substrates and catalysts. The work presented here focuses on the growth and microstructure of Ge and PbSe NWs. Ge NWs, while not a prime candidate for single-component solar cells, offer interesting opportunities for lattice matching in multijunction devices and as templates for novel architectures. Epitaxial Ge NWs are readily grown at low temperatures on Si substrates [3], so are easily integrated into current fabrication processes. $\mathrm{PbSe}$ is of interest for multi-exciton generation (MEG) devices and solar cells with PbSe quantum dots have been demonstrated. However, NWs provide a better morphology for building connected structures.

Epitaxial Ge NWs grow vertically on (111) Ge or Si substrates, with Au nanoparticles acting as a catalyst [3]. However the NW yield and size distribution is consistently better on Ge than on Si. We have found that using porous Si as the substrate also improves the yield and uniformity; SIMS and EDS data show Ge and Au diffusion into the porous substrate (Fig.1). Cross-section TEM indicates that formation of the ternary eutectic phase at the substrate competes with binary phase melting at the Au surface, leading to embedded rather than vertical NWs (Fig.2). Porous Si diminishes this effect by diverting the ternary phase into the pores. Concomitantly, the greatly enhanced surface area provided by the pores (estimated as 900X) prevents Au nanoparticle coarsening and breakup, also leading to improved NW yield and uniformity.

We observe both VLS (vapor-liquid-solid) and dislocation-mediated growth in PbSe NWs synthesized during the same processing run [4]. PbSe has a rocksalt structure and the NWs grow in the [001] direction, exhibiting secondary branching in the [100/010] directions as catalysts redeposit on the NW. Some of the NWs propagate via a screw dislocation down the center of the wire, exhibiting chiral structures observed in SEM as a rotating branch structure (Fig. 3.) Two-beam imaging confirms that the dislocation has pure screw character. The chirality is caused by the elastic strain of the axial screw dislocation, which produces a corresponding Eshelby Twist. [5]

References

[1] L. Tsakalakos, et al. Applied Physics Letters 91 (2007) 233117.

[2] C.K. Chan, et al., Nature Nanotechnology, 3 (2008) 32.

[3] H.Adhikari, et al., Nano Lett., 6 (2006) 318.

[4] J. Zhu, et al., Nature Nanotechnology, 3 (2008) 478.

[5] J.D. Eshelby, J. Appl. Phys. 24 (1953) 176. 


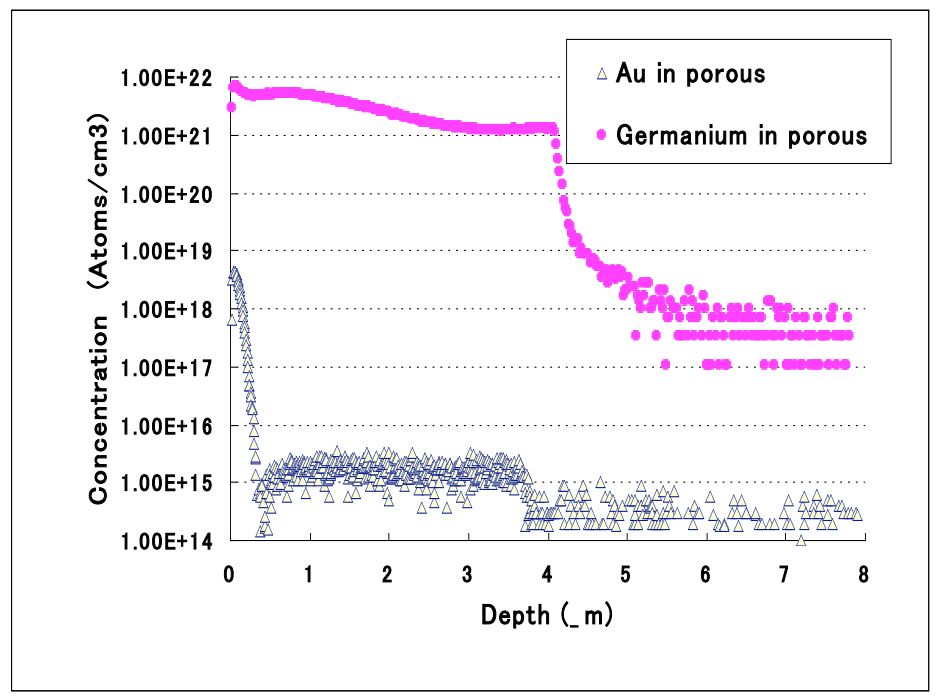

Fig. 1: SIMS data showing Ge and Au diffusion into porous Si are consistent with TEM and EDS results.

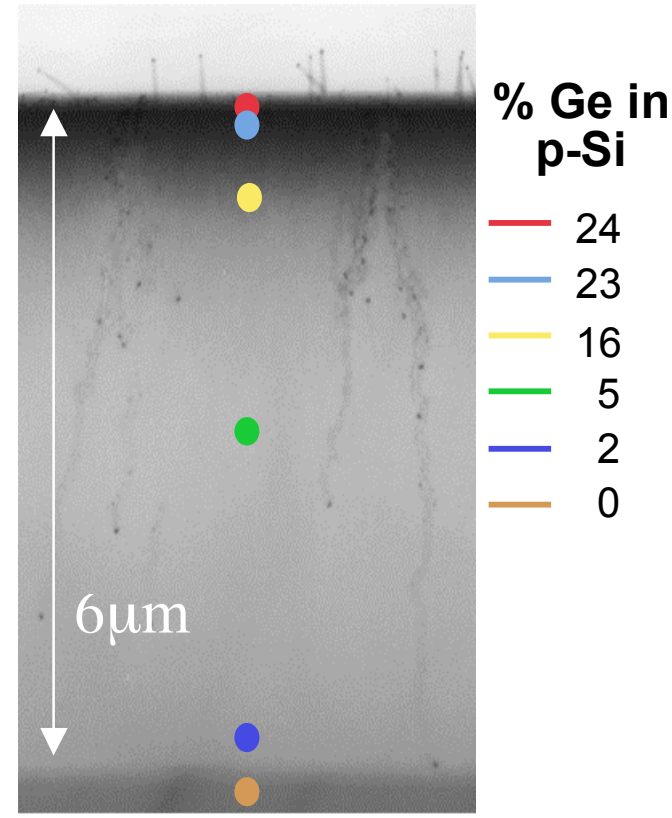

Fig. 2: In the SEM image, bright NWs grow out-ofplane; gray crooked NWs are embedded in the substrate. Cross-section TEM shows the lateral regions of embedded SiGe.

Fig. 3 SEM and TEM of chiral PbSe NWs. Twobeam imaging confirms the presence of a screw

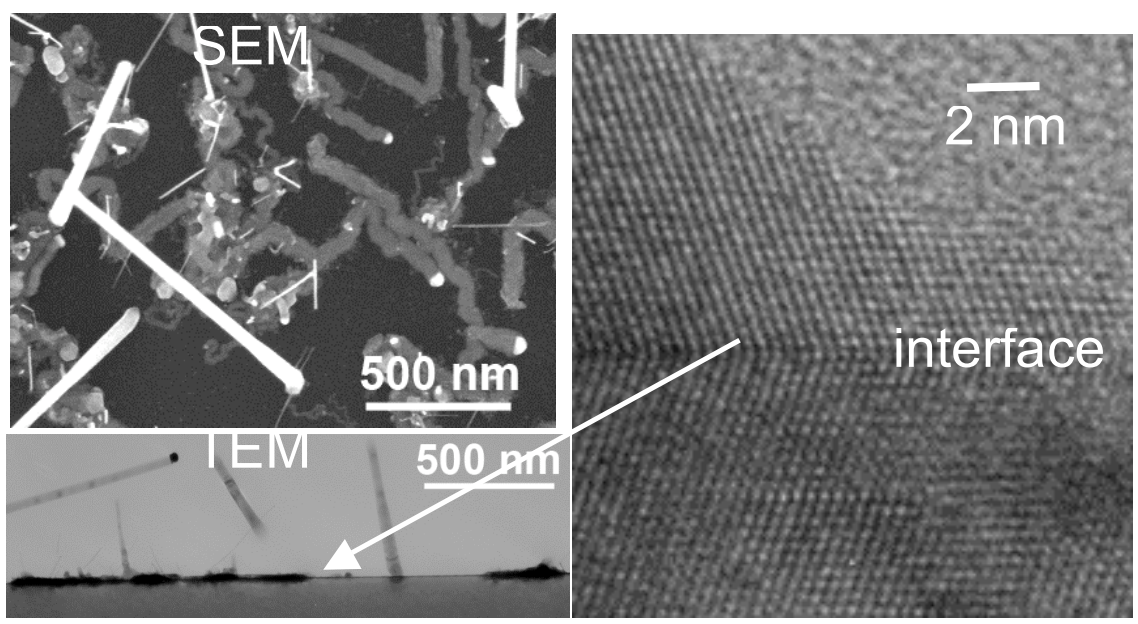
dislocation.

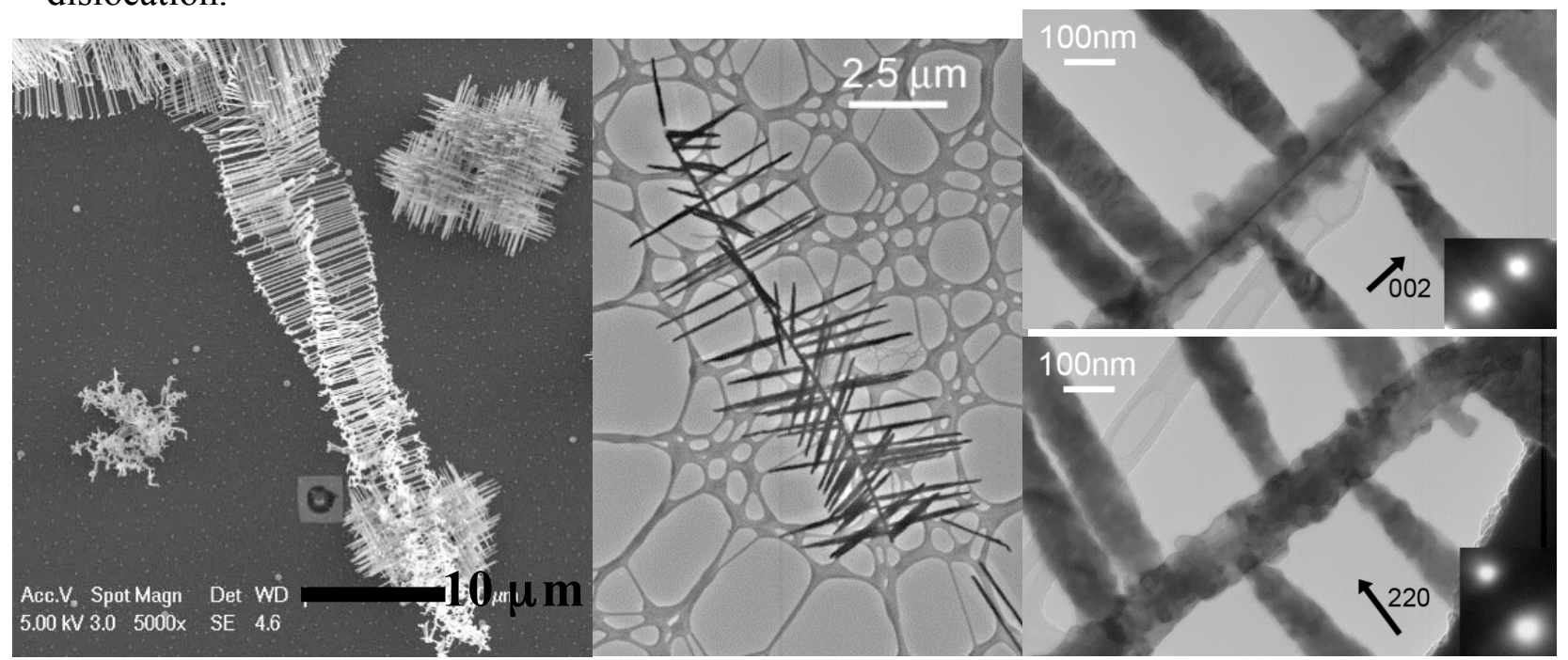

